1102 FISH OIL SUPPLEMENTATION AND PRO-INFLAMMATORY AND PRO-RESOLVING LIPID MEDIATORS IN PATIENTS WITH AND WITHOUT SYSTEMIC LUPUS ERYTHEMATOSUS

1,2May Y Choi, ${ }^{3}$ Nancy R Cook, ${ }^{1}$ Emma Stevens, ${ }^{1}$ Jack Ellrodt, ${ }^{3}$ Natalya Gomelskaya, ${ }^{3}$ Gregory Kotler, ${ }^{3}$ JoAnn E Manson, ${ }^{4}$ Jessica Lasky-Su, ${ }^{1}$ Raju Tatituri, ${ }^{3}$ Samia Mora, ${ }^{3,5}$ Min Lee, ${ }^{6}$ Charles Serhan, ${ }^{1}$ Karen H Costenbader*. ${ }^{1}$ Division of Rheumatology, Inflammation and Immunity, Department of Medicine, Brigham and Women's Hospital, Harvard Medical School, Boston, MA, USA; ${ }^{2}$ Division of Rheumatology, Cumming School of Medicine, University of Calgary, Calgary, Alberta, Canada; ${ }^{3}$ Division of Preventive Medicine, Department of Medicine, Brigham and Women's Hospital, Harvard Medical School, Boston, MA, USA; Division of Cardiovascular Medicine, Brigham and Women's Hospital and Harvard Medical School, Boston, MA, USA; Center for Lipid Metabolomics, Brigham and Women's Hospital and Harvard Medical School, Boston, MA, USA; ${ }^{4}$ Systems Genetics and Genomics Unit, Channing Division of Network Medicine Brigham and Women's Hospital and Harvard Medical School, Boston, Massachusetts, USA; ${ }^{5}$ Department of Epidemiology, Harvard T.H. Chan School of Public Health; ${ }^{6}$ Department of Anesthesia, Perioperative and Pain Medicine, Center for Experimental Therapeutics and Reperfusion Injury, Brigham and Women's Hospital-Harvard Medical School, Boston, Massachusetts, USA

\subsection{6/lupus-2021-lupus21century.45}

Background Omega-3 fatty acid-derived 'specialized pro-resolving mediators' (SPM) are low-abundance lipid mediators (LM) central to inflammation resolution. We investigated whether fish oil (FO) supplementation was associated with pro-inflammatory and pro-resolving LM in patients with SLE compared to matched controls.

Methods Within the Mass General Brigham Biobank, we identified 16 patients with SLE taking FO, who were matched by age, sex, and race to 16 patients without SLE taking FO. Another 16 patients with SLE not taking FO were matched on the same factors to 16 non-SLE patients not taking FO. Demographic and clinical data were obtained by medical record review. Targeted liquid chromatography-tandem spectroscopy was performed on plasma to quantify 27 omega3 derived LM, identified with $>6$ diagnostic ions by tandem mass spectrometry (MS-MS). Multivariable linear analyses examined the associations of SLE, FO, and their interactions with LM levels (log-transformed to improve normality), adjusting for smoking status, body mass index and medications. In SLE case-only analyses, we additionally adjusted for C-reactive protein or erythrocyte sedimentation rate (normal/elevated), anti-double stranded DNA (dsDNA positive/negative), C3 and C4 (normal/low), and presence of lupus nephritis. We adjusted for multiple comparisons using a False Discovery Rate (FDR) with a cut-off of 0.05 . For missing data, we used multiple imputation.

Results Among SLE patients, lower levels of arachidonic acid (AA) and most (60\%) of its proinflammatory derivatives were observed in those taking vs. not taking FO, whereas, among the controls, higher levels of AA and all of its pro-inflammatory derivatives were observed in those taking vs. not taking FO (figure 1). However, after adjustment for multiple comparisons, there were no significant differences for any LM between SLE compared to matched controls, taking or not taking FO. Among controls, taking FO was associated with higher levels of eicosapentaenoic acid (adjusted $\beta$ coefficient 0.67 (95\% CI: 0.28-1.07), FDR = 0.04). Taking FO was not associated with SPM levels among SLE patients even after adjusting for markers of disease activity. The interaction between SLE and FO was not statistically significant.

Conclusions In this cross-sectional study, FO supplementation among SLE patients was not significantly associated with higher levels of several pro-resolving SPMs. This may be related to a higher level of inflammatory burden in SLE patients at baseline, reduced ability to biosynthesize SPMs, or failure to take regular and adequate doses of FO. As FO preparations and doses were not controlled in this observational study, further larger controlled studies should pursue these observations.

\section{PERFLUOROALKYL SUBSTANCES AND COMMUNITY VULNERABILITY: ASSOCIATIONS WITH LUPUS-RELATED AUTOANTIBODIES AND DISEASE}

Emily L Vara, Dulaney A Wilson, John L Pearce, Jim C Oates, Diane L Kamen*. Medical University of South Carolina, USA

\subsection{6/lupus-2021-lupus21century.46}

Background Perfluoroalkyl substances (PFAS) are a class of persistent organic pollutants found in nonstick products, water repellant fabrics, fire-retardant foams, and food packaging.

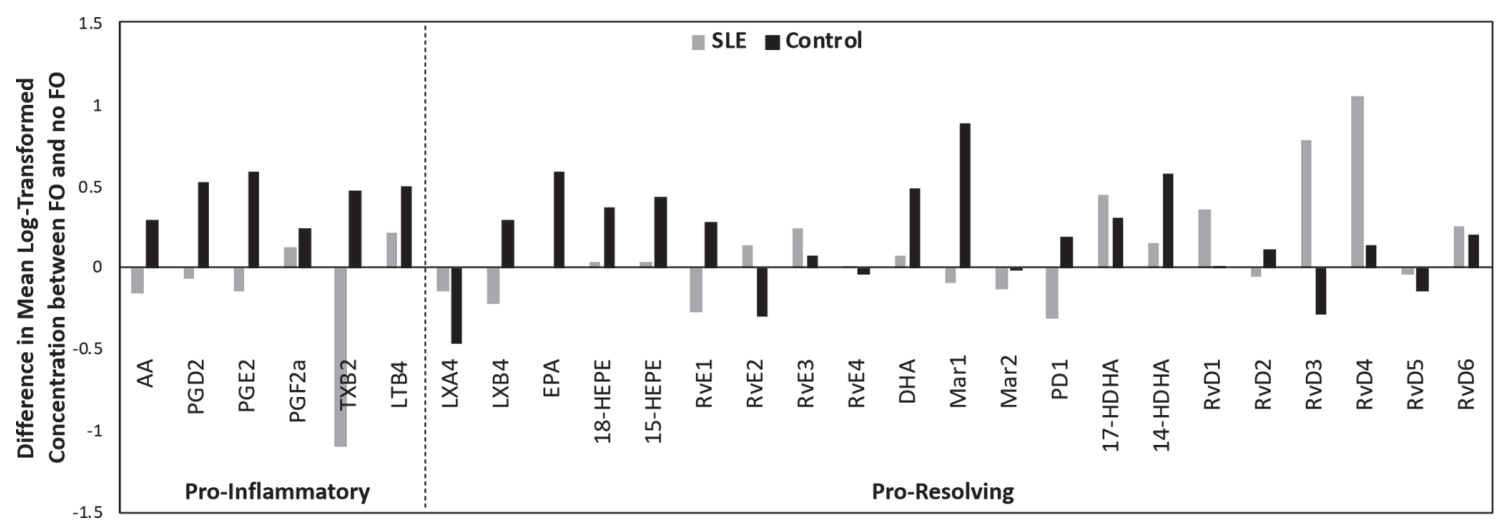

Abstract 1102 Figure 1 Difference in mean log-transformed Omega-3 fatty acid derived lipid concentration between taking vs. not taking fish oil supplementation among SLE and controls

No statistically significant differences were found after adjustment for multiple comparisons was performed using false discovery rate of 0.05 . Abbreviations: LTB4, Leukotriene B4; LXA4, Lipoxin A4; LXB4, Lipoxin B4; MaR1-2, maresin 1-2; PGD2, Prostaglandin D2; PGE2, Prostaglandin E2; PGF2a, Prostaglandin F2 alpha; TXB2, Thromboxane B2; PD1, protectin D1; RvD1-6, resolvin D-6; RvE1-4, resolvin E1-4; 14-HDHA, 14-hydroxydocosahexaenoic acid; 15-HEPE, 15-hydroxyeicosapentaenoic acid; 17-HDHA, 17hydroxy-docosahexaenoic acid; 18-HEPE, 18-hydroxyeicosapentaenoic acid. 